\title{
Cardiac Tamponade in COVID-19 Patients: Management and Outcomes
}

\author{
Hazim Hakmi $^{1}$, Amir Sohail ${ }^{1}$, Collin Brathwaite ${ }^{1}$, Ray Beevash ${ }^{1}$, and Sunil Abrol ${ }^{1}$ \\ ${ }^{1}$ NYU Langone Health
}

June 23, 2020

\begin{abstract}
Importance: Cardiac tamponade requiring emergent intervention is a possible complication of COVID-19 infection. Favorable clinical outcomes are possible if timely management and drainage are performed, unless ventricular failure develops. Observations: Cardiac tamponade in COVID-19, based on the limited reported cases, seems to be more common among middle-aged men. Prognosis is worse amongst patients with concomitant ventricular failure. Design and methods: This is a case series of three COVID-19 patients complicated by cardiac tamponade, requiring surgical intervention at a single institution in New York. Interventions: Pericardial window, Pericardiocentesis Outcomes: One patient had recurrence of cardiac tamponade with hemorrhagic component but fully recovered and was discharged home. Two patients developed cardiac tamponade with concomitant biventricular failure, resulting in death. Conclusions and Relevance: Cardiac Tamponade with possible concomitant biventricular failure can develop in COVID-19 patients; incidence seems to be highest at the point of marked inflammatory response. Concomitant ventricular failure seems to be a predictor of poor prognosis.
\end{abstract}

\section{MANUSCRIPT}

Introduction:

The World Health Organization declared the Coronavirus disease 2019 (COVID-19) outbreak as a pandemic on March 11, 2020. With its rapid spread across the globe, physicians have encountered a broad spectrum of early and late complications - mostly resulting from the underlying inflammatory process - including acute respiratory distress syndrome (ARDS), acute kidney injury (AKI), and secondary infection.

Mortality among hospitalized COVID-19 patients has been reported to be as high as $17 \%$, with the figure being $20.5 \%$ in patients undergoing elective surgeries.

There is paucity of data regarding the cardiovascular effects of COVID-19. Arrhythmias, myocarditis, hypercoagulability, congestive heart failure, and, surprisingly, two cases of cardiac tamponade physiology, both resulting from hemorrhagic pericardial effusion, have been reported. We further report three cases of COVID-19 complicated by cardiac tamponade requiring emergent surgical intervention.

\section{Case Series:}

Case 1: A 48-year-old Hispanic male with a past medical history of type 2 diabetes mellitus, morbid obesity (BMI $39.5 \mathrm{~kg} / \mathrm{m}^{2}$ ), with normal cardiac function and a known small pericardial effusion (not amenable to pericardiocentesis), presented with complaints of dyspnea and fatigue for one month, and acute deterioration for the past 24 hours. Nasopharyngeal swab was positive for COVID-19, serum troponins were within normal range, and arterial blood gas (ABG) analysis showed a $\mathrm{pH}$ of 7.26 , partial pressure of carbon dioxide (pCO2) of $83 \mathrm{mmHg}$, and partial pressure of oxygen $(\mathrm{pO} 2)$ of $187 \mathrm{mmHg}$. Chest x-ray demonstrated clear lung fields and a large cardiac silhouette. Echocardiogram showed a moderate-to-large pericardial effusion with tamponade physiology (Figure 1). 
He underwent emergent percutaneous drainage of $1,500 \mathrm{~mL}$ of clear pericardial fluid using a pigtail catheter. On post-operative day (POD) 8, the patient developed acute cardiovascular decompensation. An echocardiogram showed a large pericardial fluid collection with signs of obstructive shock (Figure 2). He underwent a sub-xyphoid pericardial drainage of $900 \mathrm{~mL}$ of sanguineous fluid. Postoperative course was unremarkable, and patient was discharged 10 days after the second procedure in stable condition with drop in his inflammatory markers after Pericardiocentesis (Table 2).

Case 2: A 56-year-old Hispanic overweight (BMI of $27.1 \mathrm{~kg} / \mathrm{m}^{2}$ ) male with no significant medical history presented with a one-week history of cough, chest pain, fever and chills. Laboratory investigations showed a serum troponin-I level of $1.2 \mathrm{ng} / \mathrm{dl}$ and nasopharyngeal swab confirmed COVID-19 infection. Over the next few hours, the patient developed progressive hypotension. Echocardiogram showed a large pericardial effusion with tamponade physiology (No images recorded in system, as it was an emergent bedside ECHO), and a left ventricular ejection fraction of $20 \%$. He underwent emergent sub-xyphoid drainage of $400 \mathrm{~mL}$ of serous pericardial fluid. On POD0, the patient experienced cardio-pulmonary arrest and expired. Inflammatory markers can be seen in (Table 2)

Case 3: A 55-year-old African American male with a past medical history of hypertension and obesity (BMI of $30.5 \mathrm{~kg} / \mathrm{m}^{2}$ ) presented with two weeks of dyspnea, productive cough, myalgias, headaches, fatigue, fever, and chills. Upon initial evaluation, patient was placed on 15 liters/min of oxygen via a non-rebreather mask for hypoxia. ABG showed a $\mathrm{pH}$ of 7.46, $\mathrm{pCO} 2$ of $32 \mathrm{mmHg}$, and $\mathrm{pO} 2$ of $67 \mathrm{mmHg}$. Serum troponin-I was within normal range, serum creatinine was $2.3 \mathrm{mg} / \mathrm{d}$, and nasopharyngeal swab was positive for COVID-19. CXR showed bilateral lung opacities and a mildly enlarged cardiac silhouette. On hospital day two, the patient required endotracheal intubation and mechanical ventilation for progressive hypoxia, and subsequently, on hospital day 5, required Veno-Venous Extra Corporeal Membrane Oxygenation (ECMO). Laboratory investigations during hospitalization are seen in (Table 3). On hospital day 7, the patient developed pulseless electrical activity (PEA) cardiac arrest. A bedside echocardiogram revealed a large pericardial effusion with tamponade physiology, and $800 \mathrm{~mL}$ of sanguineous fluid was drained percutaneously, with return of spontaneous circulation. On hospital day 8 , due to increasing vasopressor and inotropic requirements, a repeat echocardiogram was performed which demonstrated biventricular failure, likely from COVID-related myocarditis (Figure 3). Further aggressive management was deemed futile, and all interventions were withdrawn per family's wishes; the patient underwent ECMO decannulation and was pronounced dead.

\section{Interventions:}

Case 1 received azithromycin and hydroxychloroquine, intravenous hydrocortisone, colchicine, and zinc. Pericardiocentesis and pericardial window were performed on hospital days 1 and 7 , respectively. He received therapeutic anti-coagulation with enoxaparin for the first 7 days due to elevated D-dimer level, which was transitioned to deep venous thrombosis (DVT) prophylactic dose enoxaparin for the following 9 days after development of sanguineous pericardial tamponade.

Case 2 received intravenous hydrocortisone, hydroxychloroquine and azithromycin, and required pericardial window on hospital day 1.

Case 3 received azithromycin, hydroxychloroquine, and tocilizumab twice (before and after ECMO). The patient received ceftriaxone and doxycycline initially and was subsequently transitioned to cefepime. He required ECMO and pericardiocentesis on hospital days 5 and 7 , respectively, as well as mechanical venti-

lation for 6 days. DVT prophylactic dose of unfractionated heparin was administered for the first 5 days of hospitalization, followed by therapeutic dose for the next 4 days.

\section{Outcomes:}

Case 1 developed cardiac tamponade without myocardial dysfunction. He developed recurrent cardiac tamponade that was drained, resulting in resolution of symptoms until discharge in stable condition.

Case 2 developed severe biventricular cardiac failure at the time of development of tamponade, resulting in immediate death. 
Case 3 was admitted with AKI and worsening biventricular cardiac dysfunction, prior to pericardiocentesis that resulted in a progressive decline in hemodynamic status, ultimately leading to withdrawal of care.

\section{Discussion:}

COVID-19 was confirmed in a cluster of patients by the Chinese health authorities on January 7, 2020. The first case in the United States was reported on January 20, 2020. With its continued spread globally, a range of complications affecting almost every organ system have been reported.

Cardiac tamponade is most commonly idiopathic, with a smaller subset of cases resulting from infectious causes. It can be due to collection of transudate, exudate or blood in the pericardium. Viral pericarditis is usually characterized by a gradual accumulation of transudate. Interestingly, in COVID-19 patients with cardiac tamponade, some develop sanguineous fluid collection while others have an exudative or transudative pericardial fluid, as evidenced in our patients.

The pathophysiology of COVID-19 cardiac tamponade is possibly a result of the marked systemic inflammatory response to the virus, leading to myocarditis and pericarditis. Myocardial ischemia, as evidenced by elevated or up-trending troponin levels, can also be explained by the hyper-coagulability (resulting from endothelial damage and the inflammatory response) in these patients. Thus, prophylactic use of corticosteroids and therapeutic anti-coagulation have been proposed in COVID-19 patients.

Cardiac tamponade should be suspected in COVID-19 patients with progressive hemodynamic compromise, especially if the usual constellation of physical exam findings associated with cardiac tamponade is present. An echocardiogram showing late diastolic collapse of the right atrium and early diastolic collapse of the right ventricle (occurring when the intra-pericardial pressure exceeds intracavitary pressure) is diagnostic. Of note, CXR does not demonstrate cardiac enlargement until at least $200 \mathrm{~mL}$ of pericardial fluid has accumulated and thus has low sensitivity.

After pericardial fluid drainage management is largely supportive, and recurrent cardiac tamponade is possible. While the use of therapeutic anticoagulation has been shown to improve prognosis in severe COVID-19 patients the development of sanguineous cardiac tamponade (as seen in Case 1) may point towards increased risks of therapeutic anticoagulation after initial drainage. However, given the documented benefits of therapeutic anticoagulation in COVID-19 patients, particularly in patients with elevated D-dimers, it might be judicious to resume anti-coagulation 12 hours post-operatively, with a high index of suspicion for rebleeding in case of progressive worsening of hemodynamic status. The role of NSAIDs in this subpopulation is yet to be determined. High suspicion for recurrence of cardiac tamponade is warranted as risk of recurrence is as high as $20 \%$.

While current data on cardiac tamponade, with possible concomitant biventricular failure, in COVID-19 patients are limited, we hypothesize that short-term prognosis in this subpopulation is primarily dependent upon ventricular function at the time of development of tamponade. In our case series, the two patients with cardiac tamponade with concomitant biventricular failure (Cases 2 and 3) experienced rapid deterioration leading to death, while the patient with preserved ventricular function (Case 1) survived. However, the observed ventricular dysfunction - possibly stress cardiomyopathy or cytokine-related myocardial dysfunction - may just be a manifestation of the overall severity of inflammatory response and the associated fulminant cytokine release (Tables 1-3). Further, the long-term prognosis, sequelae and predictors of survival in COVID19 patients developing transient myocarditis or cardiac tamponade remain yet to be

Importantly, surgical intervention and drainage of pericardial fluid in COVID-19 patients, while allowing for rapid relief from tamponade physiology, is associated with intra- and post-operative risks. Anesthesia, mechanical ventilation, and use of vasopressors and inotropic medications may contribute to long-term pulmonary and cardiovascular compromise and increased mortality in COVID-19 patients. Thus, pericardiocentesis, with its minimal risk of anesthesia, may be preferable in this subpopulation.

\section{Limitations:}


Our study is limited by the small sample size, and inability to test pericardial fluid for COVID-19 antibodies and fluid composition.

\section{Conclusion:}

Cardiac tamponade is a rare complication of COVID-19 infection. Physicians must be cognizant of this possibility in patients with cardiovascular decompensation. Ultrasonography can aid in rapid diagnosis, and drainage of pericardial fluid and can result in clinical improvement. Short-term prognosis appears to depend upon ventricular function. Role of corticosteroids, NSAIDs, and immune modulators remains unclear in this subgroup of COVID-19 patients, but may have a role in preventing the development of ventricular failure caused by the marked inflammatory response and stress cardiomyopathy.

\section{Figures:}

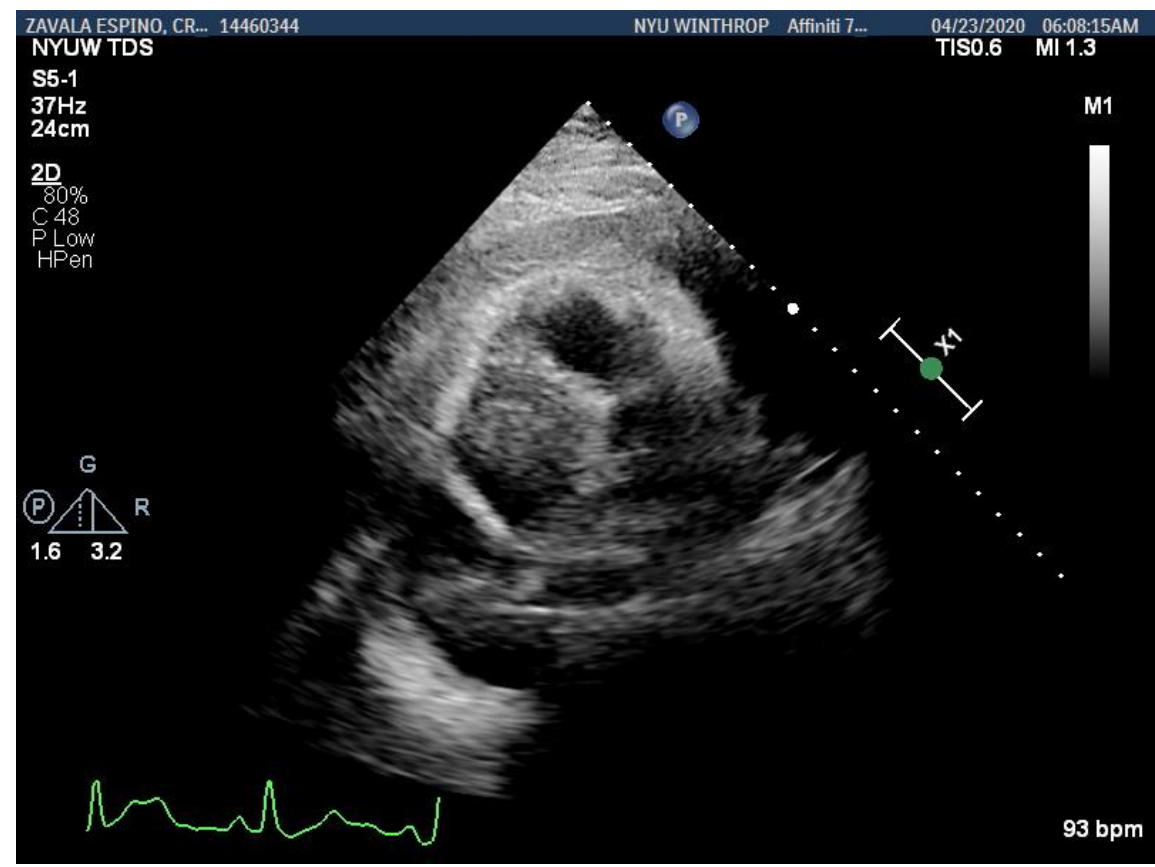



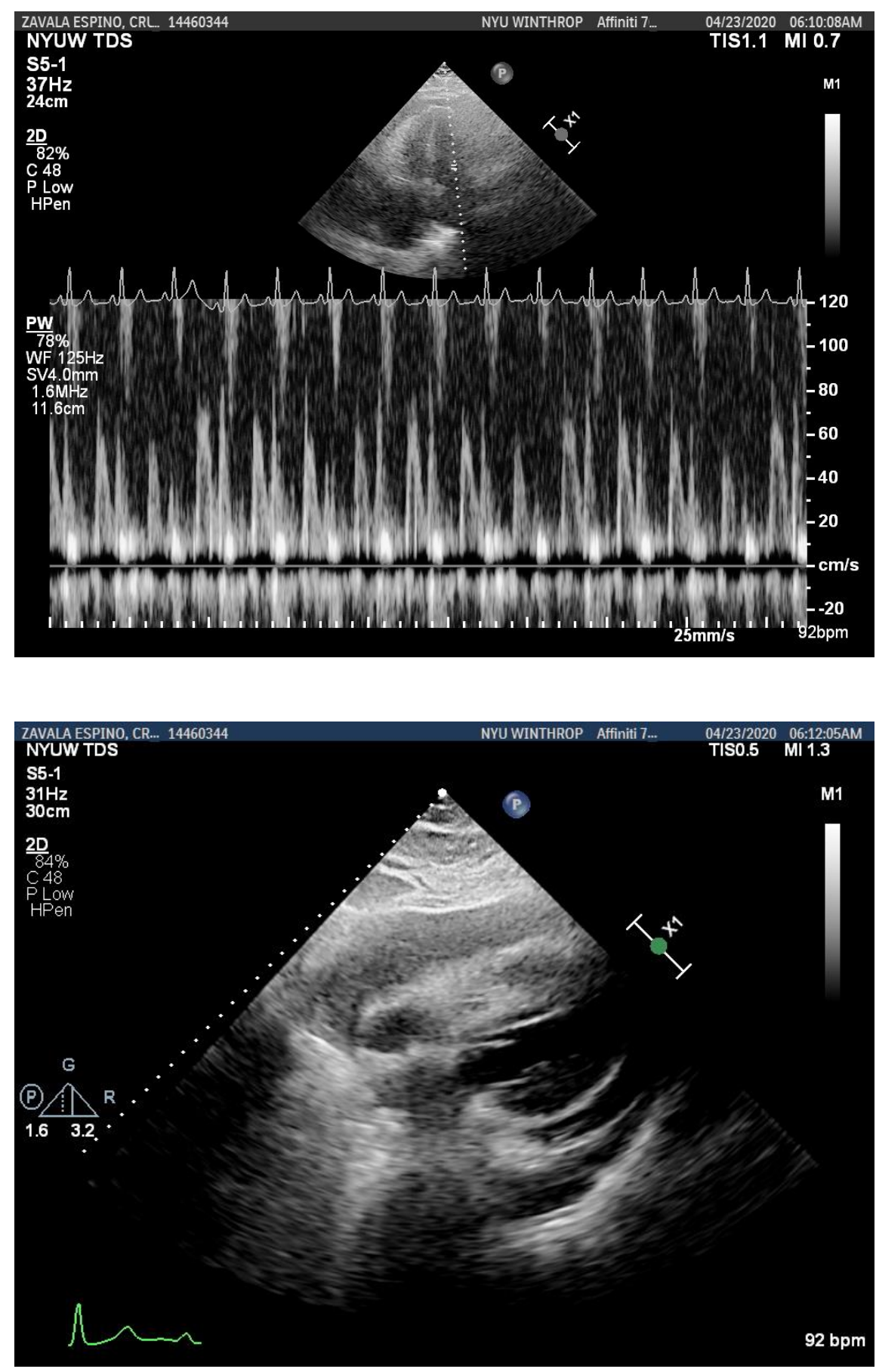

Figure 1: A) Top Left, Subcostal view: There is a large circumferential pericardial effusion

B) Top right, Continuous wave Doppler through the mitral valve in the four chamber apical view: This image shows significant $(>30 \%)$ respiratory variation in mitral inflow velocity. C) Bottom, Subcostal view: There is a large circumferential pericardial effusion with visible partial collapse of the right ventricle and right atrium. 


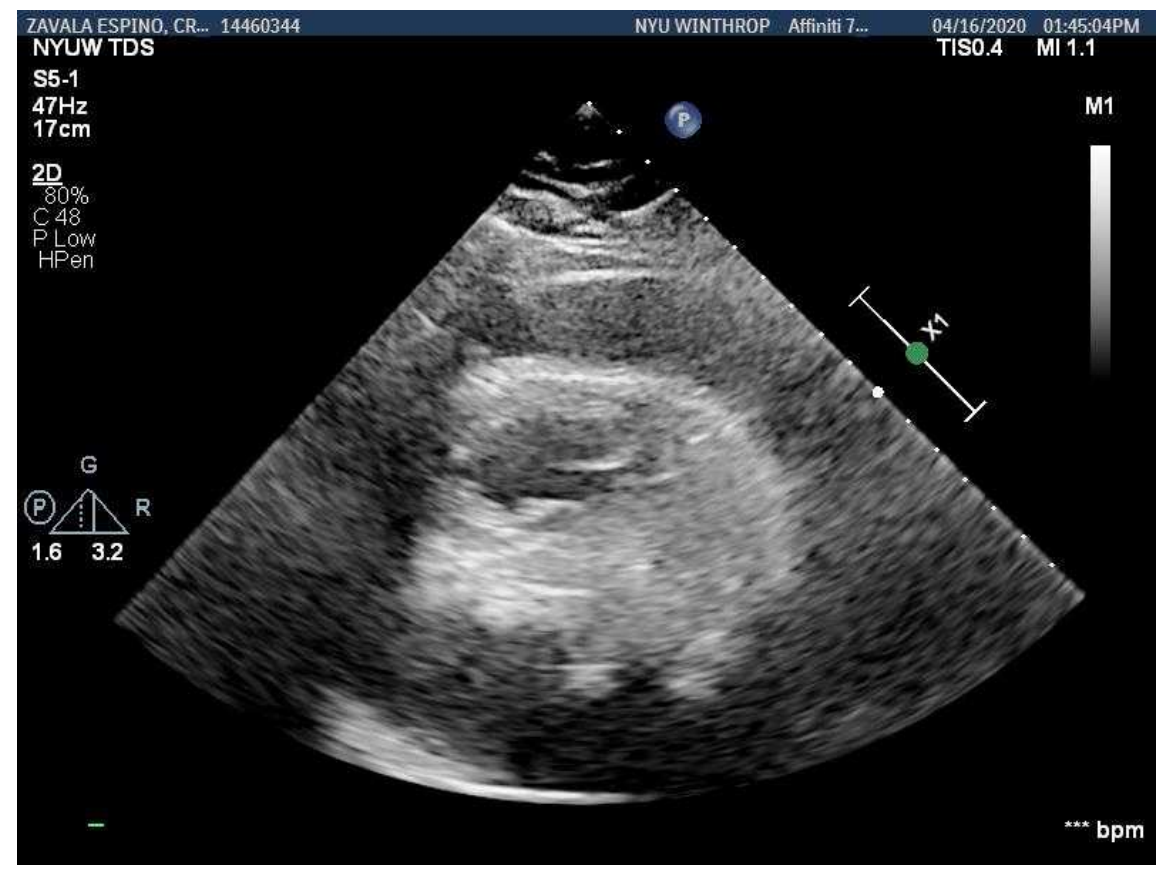

Figure 2: Subcostal view: There is a large circumferential pericardial effusion.

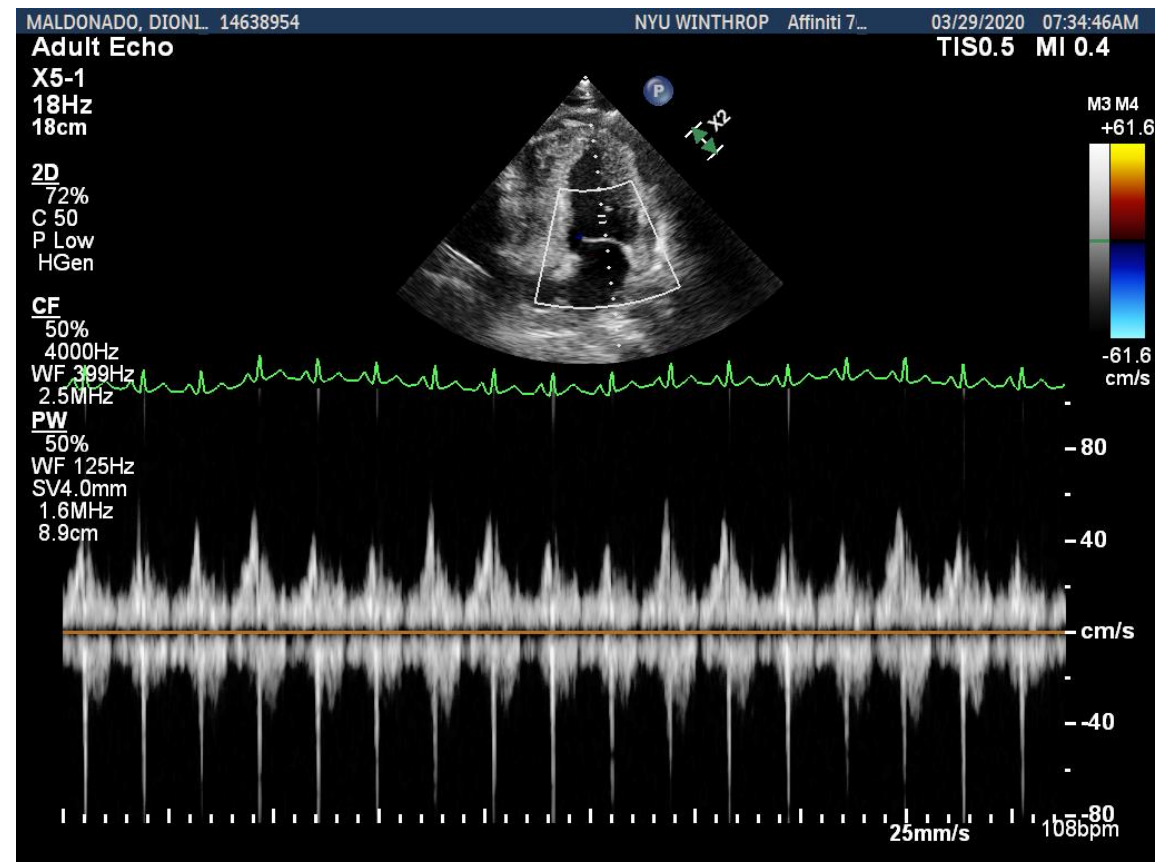



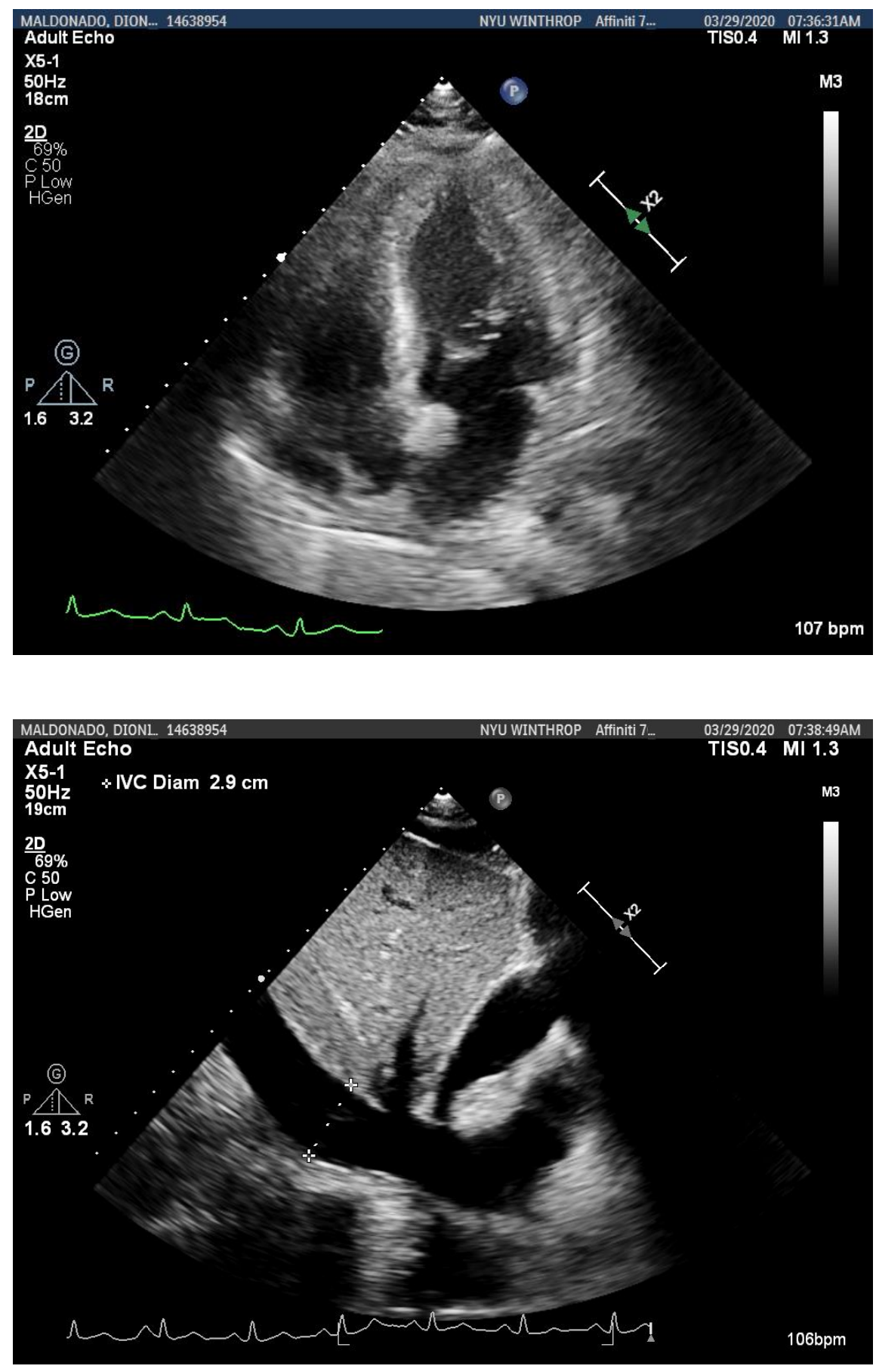

Figure 3: A) Top Left, Continuous wave Doppler through the mitral valve in the two chamber apical view: This image shows significant $(>30 \%)$ respiratory variation in mitral inflow velocity.

B) Top Right, Apical four chamber view: There is a moderate size effusion adjacent to the right atrium with partial right atrial collapse. C) Bottom, Subcostal view: The inferior vena cava is plethoric measuring 2.9 $\mathrm{cm}$.

\section{Tables:}




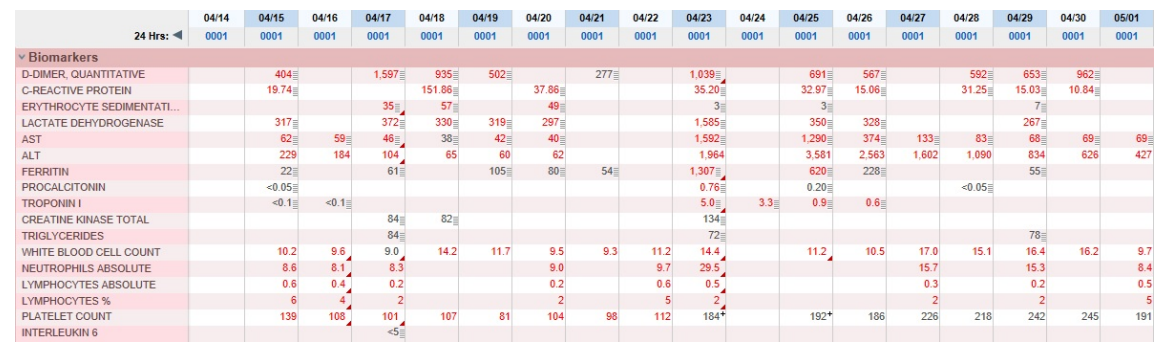

Table 1: Pt 1 Inflammatory Markers during admission.

\begin{tabular}{|c|c|c|c|c|c|}
\hline & $03 / 26$ & $03 / 27$ & $03 / 28$ & $03 / 29$ & $03 / 30$ \\
\hline 24 Hrs: & 0001 & 0001 & 0001 & 0001 & 0001 \\
\hline \multicolumn{6}{|l|}{$\checkmark$ Biomarkers } \\
\hline D-DIMER, QUANTITATIVE & & & & $<202 \equiv$ & \\
\hline C-REACTIVE PROTEIN & & & & $24.90 \equiv$ & \\
\hline LACTATE DEHYDROGENASE & & & & $240 \equiv$ & \\
\hline AST & & & $26 \equiv$ & $55 \equiv$ & $24=$ \\
\hline ALT & & & 26 & 65 & $12=$ \\
\hline FERRITIN & & & & $1,008 \equiv$ & \\
\hline PROCALCITONIN & & & & $0.08=$ & \\
\hline TROPONIN I & & & 1.2 非 & $0.5 \overline{\overline{\underline{\underline{A}}}}$ & \\
\hline CREATINE KINASE TOTAL & & & & $110 \equiv$ & \\
\hline WHITE BLOOD CELL COUNT & & & 5.5 & 4.5 & \\
\hline NEUTROPHILS ABSOLUTE & & & 4.5 & 3.6 & \\
\hline LYMPHOCYTES ABSOLUTE & & & 0.5 & 0.5 & \\
\hline LYMPHOCYTES \% & & & 9 & 12 & \\
\hline PLATELET COUNT & & & 95 & 82 & \\
\hline
\end{tabular}

Table 2: Pt 2 Inflammatory Markers during admission.

\begin{tabular}{|c|c|c|c|c|c|c|c|c|c|c|c|}
\hline & $03 / 23$ & $03 / 24$ & $03 / 25$ & $03 / 26$ & $03 / 27$ & $03 / 28$ & 03/29 & $03 / 30$ & $03 / 31$ & $04 / 01$ & $04 / 02$ \\
\hline $24 \mathrm{Hrs:}$ & 0001 & 0001 & 0001 & 0001 & 0001 & 0001 & 0001 & 0001 & 0001 & 0001 & 0001 \\
\hline \multicolumn{12}{|l|}{$\checkmark$ Biomarkers } \\
\hline D-DIMER, QUANTITATIVE & & & & & 15,212 夆 & $11,119 \bar{\equiv}$ & & 43,874 夆 & & & \\
\hline C-REACTIVE PROTEIN & & & & & $205.23 \equiv$ & 324.44文 & & 363.53 赔 & $230.99 \equiv$ & & \\
\hline ERYTHROCYTE SEDIMENTATI... & & & & & 66 夆 & $62 \equiv$ & & $65 \overline{\underline{1}}$ & & & \\
\hline LACTATE DEHYDROGENASE & & & Specim... 垟 & & $2,445=$ & 2,303플 & & 1,997 & 1,941立 & & \\
\hline AST & & & & & Speci...立 & 143” & & 66 & $262 \equiv_{4}$ & & $>6,600 \equiv$ \\
\hline ALT & & & & & $90^{\circ}$ & 77 & & $55 \equiv$ & 133 & & 3,306 \\
\hline FERRITIN & & & & & 12,310 & $6,880 \equiv$ & & $4,578 \equiv$ & & & \\
\hline PROCALCITONIN & & & & 0.94咅 & & & & & & & \\
\hline TROPONINI & & & $0.4 \equiv$ & & $0.3 \bar{\equiv}$ & & & & 0.4 寻 & & \\
\hline CREATINE KINASE TOTAL & & & 3,744立 & & 3,158砉 & 6.547咅 & 6,875 靖 & 4,417 吾 & $1,218 \equiv$ & & 1,411 \\
\hline TRIGLYCERIDES & & & & & & & & & 479 & & \\
\hline WHITE BLOOD CELL COUNT & & & 10.7 & 12.3 & 14.1 & 15.6 & 22.0 & $27.6 \mathrm{~A}$ & 27.9 . & 28.2. & 31.4 \\
\hline LYMPHOCYTE ABSOLUTE CAL... & & & 1.7 & 1.6 & 0.8 & 0.5 & 0.9 & 0.7 & & & \\
\hline LYMPHOCYTES \% & & & 13 & 12 & 6 & 3 & 4 & 3 & & & \\
\hline PLATELET COUNT & & & 255 & 236 & 231 & 228 & 200 & 141. & 92. & ${ }^{60}{ }_{4}$ & 51 \\
\hline INTERFERON GAMMA & & & & & & $<5$ & & $<5$ & & $<5^{+}$ & \\
\hline INTERLEUKIN 10 & & & & & & 44 & & 39 & & 128 & \\
\hline INTERLEUKIN 12 & & & & & & $<5$ & & $<5$ & & $<5^{+}$ & \\
\hline INTERLEUKIN 13 & & & & & & $<5$ & & $<5$ & & $<5^{+}$ & \\
\hline INTERLEUKIN-1 BETA & & & & & & $<5$ & & $<5$ & & $<5^{+}$ & \\
\hline Interleukin 2 Receptor (CD25), Sol... & & & & & & 524 & & See no... 三 & & $1,578^{+}$ & \\
\hline INTERLEUKIN 2 & & & & & & $<5$ & & $<5$ & & $<5^{+}$ & \\
\hline INTERLEUKIN 4 & & & & & & $<5$ & & $<5$ & & $<5^{+}$ & \\
\hline INTERLEUKIN 5 & & & & & & $<5$ & & $<5$ & & $<5^{+}$ & \\
\hline INTERLEUKIN 6 & & & & & & 130 & & See no...音 & See no...兰 & See $n \ldots \underline{\Xi}^{+}$ & \\
\hline INTERLEUKIN 8 & & & & & & $<5$ & & $<5$ & & 35 & \\
\hline Tumor Necrosis Factor-Alpha & & & & & & $<5 \equiv$ & & $<5$ & & 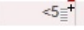 & \\
\hline
\end{tabular}


Table 3: Pt 3 Inflammatory Markers during admission.

\section{Hosted file}

Figure 1.docx available at https://authorea.com/users/335970/articles/461777-cardiactamponade-in-covid-19-patients-management-and-outcomes

\section{Hosted file}

Figure 2.docx available at https://authorea.com/users/335970/articles/461777-cardiactamponade-in-covid-19-patients-management-and-outcomes

\section{Hosted file}

Figure 3.docx available at https://authorea.com/users/335970/articles/461777-cardiactamponade-in-covid-19-patients-management-and-outcomes

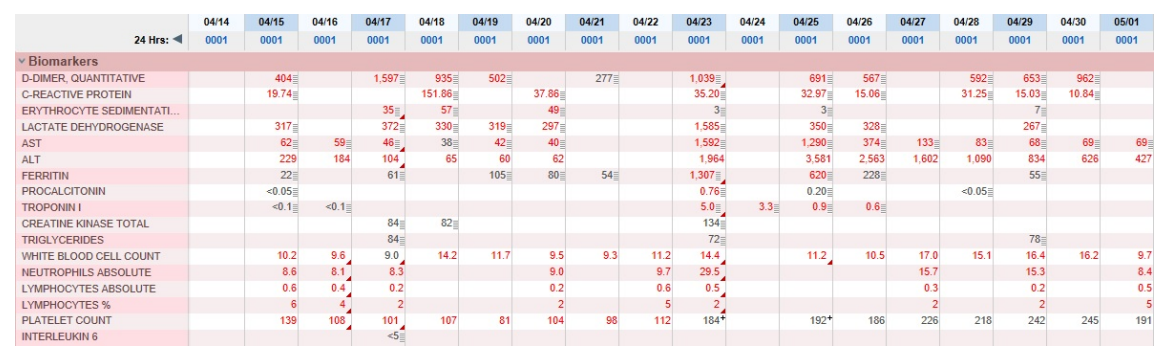

\begin{tabular}{|c|c|c|c|c|c|}
\hline & $03 / 26$ & 03/27 & $03 / 28$ & $03 / 29$ & $03 / 30$ \\
\hline 24 Hrs: & 0001 & 0001 & 0001 & 0001 & 0001 \\
\hline \multicolumn{6}{|l|}{$\checkmark$ Biomarkers } \\
\hline D-DIMER, QUANTITATIVE & & & & $<202$ 三 & \\
\hline C-REACTIVE PROTEIN & & & & $24.90 \equiv$ & \\
\hline LACTATE DEHYDROGENASE & & & & $240 \equiv$ & \\
\hline AST & & & $26 \equiv$ & $55 \equiv$ & $24 \equiv$ \\
\hline ALT & & & 26 & 65 & $12 \equiv$ \\
\hline FERRITIN & & & & $1,008 \equiv$ & \\
\hline PROCALCITONIN & & & & $0.08 \bar{\equiv}$ & \\
\hline TROPONIN I & & & $1.2 \bar{\equiv}$ & $0.5 \equiv$ & \\
\hline CREATINE KINASE TOTAL & & & & $110 \equiv$ & \\
\hline WHITE BLOOD CELL COUNT & & & 5.5 & 4.5 & \\
\hline NEUTROPHILS ABSOLUTE & & & 4.5 & 3.6 & \\
\hline LYMPHOCYTES ABSOLUTE & & & 0.5 & 0.5 & \\
\hline LYMPHOCYTES \% & & & 9 & 12 & \\
\hline PLATELET COUNT & & & 95 & 82 & \\
\hline
\end{tabular}




\begin{tabular}{|c|c|c|c|c|c|c|c|c|c|c|c|}
\hline & $03 / 23$ & $03 / 24$ & $03 / 25$ & $03 / 26$ & $03 / 27$ & $03 / 28$ & $03 / 29$ & $03 / 30$ & $03 / 31$ & $04 / 01$ & $04 / 02$ \\
\hline $24 \mathrm{Hrs}:$ & 0001 & 0001 & 0001 & 0001 & 0001 & 0001 & 0001 & 0001 & 0001 & 0001 & 0001 \\
\hline \multicolumn{12}{|l|}{$\checkmark$ Biomarkers } \\
\hline D-DIMER, QUANTITATIVE & & & & & 15,212 夆 & 11,119 严 & & $43,874 \bar{\equiv}$ & & & \\
\hline C-REACTIVE PROTEIN & & & & & 205.23 & 324.44 翟 & & 363.53 位 & $230.99=$ & & \\
\hline ERYTHROCYTE SEDIMENTATI... & & & & & $66 \equiv$ & 62 & & $65 \equiv$ & & & \\
\hline LACTATE DEHYDROGENASE & & & Specim...言 & & 2,445 夆 & 2,303플 & & $1,997 \overline{\bar{\equiv}}$ & 1,941 立 & & \\
\hline AST & & & & & 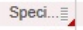 & 143! & & 66 & $262 \equiv$ & & $>6,600 \equiv$ \\
\hline ALT & & & & & 90 & 77 & & $55 \bar{\equiv}$ & $133^{4}$ & & 3,306 \\
\hline FERRITIN & & & & & 12,310 & $6,880 \equiv$ & & $4,578 \equiv$ & & & \\
\hline PROCALCITONIN & & & & 0.94 击 & & & & & & & \\
\hline TROPONINI & & & $0.4 \equiv$ & & 0.3 昰 & & & & 0.4 寻 & & \\
\hline CREATINE KINASE TOTAL & & & 3,744文 & & 3,158夆 & $6,547 \equiv$ & 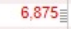 & 4,417文 & $1,218 \equiv$ & & 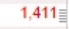 \\
\hline TRIGLYCERIDES & & & & & & & & & $479 \equiv$ & & \\
\hline WHITE BLOOD CELL COUNT & & & 10.7 & 12.3 & 14.1 & 15.6 & 22.0 & 27.6 & $27.9^{\circ}$ & 28.2 & 31.4 \\
\hline LYMPHOCYTE ABSOLUTE CAL... & & & 1.7 & 1.6 & 0.8 & 0.5 & 0.9 & 0.7 & & & \\
\hline LYMPHOCYTES \% & & & 13 & 12 & 6 & 3 & 4 & 3 & & & \\
\hline PLATELET COUNT & & & 255 & 236 & 231 & 228 & 200 & 141 & 92 . & 60 & 51 \\
\hline INTERFERON GAMMA & & & & & & $<5$ & & $<5$ & & $<5^{+}$ & \\
\hline INTERLEUKIN 10 & & & & & & 44 & & 39 & & 128 & \\
\hline INTERLEUKIN 12 & & & & & & $<5$ & & $<5$ & & $<5^{4}$ & \\
\hline INTERLEUKIN 13 & & & & & & $<5$ & & $\ll 5$ & & $<5^{+}$ & \\
\hline INTERLEUKIN-1 BETA & & & & & & $<5$ & & $\ll 5$ & & $<5^{+}$ & \\
\hline Interleukin 2 Receptor (CD25), Sol... & & & & & & 524 & & See no...具 & & $1,578^{+}$ & \\
\hline INTERLEUKIN 2 & & & & & & $<5$ & & $<5$ & & $<5^{+}$ & \\
\hline INTERLEUKIN 4 & & & & & & $<5$ & & $<5$ & & $<5^{+}$ & \\
\hline INTERLEUKIN 5 & & & & & & $<5$ & & $<5$ & & $<5^{+}$ & \\
\hline INTERLEUKIN 6 & & & & & & 130 & & See no...立 & See no...亩 & See $n \ldots \Xi^{+}$ & \\
\hline INTERLEUKIN 8 & & & & & & $<5$ & & $<5$ & & 35 & \\
\hline Tumor Necrosis Factor-Alpha & & & & & & $<5$ & & $<5$ & & $<5 \pm^{+}$ & \\
\hline
\end{tabular}

